\title{
Presencia de sesgos cognitivos y afectación del clima familiar en jugadores patológicos y no patológicos de la ciudad de Bogotá
}

Iza Certuche, Daniel Andrés ${ }^{1}$.

\begin{abstract}
Resumen | Para hacer un abordaje sobre la problemática del juego patológico en la ciudad de Bogotá, se analizó una muestra de 120 personas dividida en cuatro grupos iguales, los cuales se organizaron entre jugadores de 18 a 35 años, no jugadores de 18 a 35 años, jugadores de 36 a 60 años y no jugadores de 36 a 60 años. Se buscó ver las diferencias entre los grupos teniendo en cuenta las variables de sesgos cognitivos y clima familiar. Se realizó una pregunta adicional sobre apuestas por internet para establecer el porcentaje de participación en cada grupo. Los grupos de posibles jugadores patológicos y los no jugadores, independientemente de la edad, presentaron diferencias en las variables de sesgos cognitivos y clima familiar. Los resultados apoyan la idea de que existen cambios en la regulación emocional dependiendo de la edad, lo que influye en la toma de decisiones de riesgo. El clima familiar se vio afectado en jugadores y no jugadores, esto puede obedecer a otras variables culturales. Por último, se observó un alto porcentaje de personas que realizan apuestas por Internet en todos los grupos, por lo que es necesario ampliar los estudios en esta vía.
\end{abstract}

\author{
Editado por \\ William Alejandro Jiménez-Jiménez \\ Universidad de Boyacá, Colombia. \\ Revisado por \\ Raúl Quevedo-Blasco \\ Universidad de Granada (España).
}

${ }^{1}$ Daniel Andrés Iza Certuche
$[\square]$

Psicólogo Universidad Nacional de Colombia. Candidato a Maestría en Psicología Clínica de la Universidad Nacional de Colombia. Docente Corporación Universitaria Minuto de Dios e-mail: Daniel.iza@uniminuto.edu 
Palabras Clave | Juego patológico, casinos, sesgos cognitivos, clima familiar, apuestas por internet.

\begin{abstract}
To make an approach on the problem of pathological gambling in Bogota, a sample of 120 people divided into four equal groups analyzed, groups, not players $18-35$ years old, were organized between players of 18 to 35 players of 36-60 years and 36-60 players years. It sought to see the differences between the groups considering the variables of cognitive biases and family atmosphere. An additional question about online betting was conducted to establish the percentage of participation in each group. Groups of potential pathological gamblers and non-gamblers, regardless of age, showed differences in the variables of cognitive biases and family atmosphere. The results support the idea that there are changes in emotional regulation depending on age, which influences the decision-making risk. The family atmosphere was affected players and non-players, this may be due to other cultural variables. Finally a high percentage of people doing Internet gambling in all groups was observed, so it is necessary to expand studies in this way.
\end{abstract}

Keywords | Pathological Gambling, Casinos, cognitive biases, family atmosphere, internet betting .

\title{
Introducción
}

En Colombia, según la encuesta de Calidad de Vida del DANE en el 2008, la cuarta parte de los hogares hacen algún tipo de apuestas, entre las que se encuentran la compra de loterías y/o chance. Departamentos como Valle, Antioquia, San Andrés y la Costa Atlántica destinan entre el $25 \%$ y el $35 \%$ de los ingresos mensuales a esta actividad, lo que se ve representado en una venta anual de alrededor 1,8 billones de pesos (Dane, 2008).

Con la ayuda de los medios masivos de comunicación, la industria de los juegos de azar creció vertiginosamente, al punto de convertirse en un problema social por su característica patológica. Este crecimiento presenta la tarea de estudiar las dinámicas de los jugadores de azar, observar las variables presentes y las implicaciones en todo nivel puesto que el juego patológico se 
constituye no solo en una categoría diagnóstica, sino también, en fuente de una problemática económica y social devastadora entre quienes lo padecen.

Una de las características más importantes a destacar en el juego patológico es la elevada comorbilidad con otros cuadros clínicos. Cuando una persona es adicta, lo más habitual es que en un $50 \%$ y $75 \%$ de los casos, se presente un trastorno adicional (Fernández \& López, 2005).

Para los ludópatas, la ingesta excesiva de alcohol o drogas alucinógenas, son el trastorno más relacionado, seguido de la delincuencia organizada y la violencia. Así mismo, tiene un alto impacto en adolescentes, al estar más propensos a desarrollar problemas de abuso de sustancias cuando realizan actividades en sitios de apuestas (Unwin, Davis \& De Leeuw, 2000). La ludopatía, es entonces, un grave problema que debe ser analizado cuidadosamente para determinar formas efectivas de detección y prevención, pues trae múltiples consecuencias al interior de la familia y del entorno social del sujeto.

El apostar en cualquier juego de azar como las loterías o juegos de habilidad como las cartas, no son por sí mismas conductas anormales, sino mas bien actividades recreativas por cuanto pretenden generar bienestar e interacción social. Sin embargo, de dichas actividades se pueden hacer usos anormales en función de la intensidad, de la frecuencia o de la cantidad de dinero invertido y, en último término, en función del grado de interferencia en las relaciones familiares, sociales, y laborales de las personas implicadas.

Cualquier conducta normal y placentera es susceptible de convertirse en una conducta adictiva, lo que define a esta última es que la persona pierde el control de la actividad y continúa con ella a pesar de las consecuencias adversas, supeditando el estilo de vida al mantenimiento del habito (Fernández, 1997, p. 102)

La Organización Mundial de la Salud (OMS) desde el año 1992, incluye a la ludopatía en la décima revisión de su Clasificación Internacional de Enfermedades (CIE), definiéndola como un trastorno caracterizado por la presencia de: "frecuentes y reiterados episodios de participación en juegos de apuestas, los cuales dominan la vida del enfermo en perjuicio de sus valores y obligaciones sociales, laborales, materiales y familiares" (CIE-10 1992). 
De acuerdo con el contexto mencionado, el siguiente trabajo hace una exploración de algunas particularidades en las personas que frecuentan sitios de juegos de azar como casinos y lugares de apuestas, con el fin de observar las formas en las cuales los jugadores se ven implicados emocionalmente en el juego, las relaciones entre el juego patológico y la edad en la que se encuentran los individuos y sus relaciones familiares. Por último, se intentó establecer si las personas que tienen problemas con el juego en la actualidad realizan actividades de apuestas por Internet, al ser una nueva modalidad que toma gran fuerza a nivel mundial.

\section{Distorsiones cognitivas en el juego patológico}

Desde 1970 con los revolucionarios estudios de Kahneman y Tversky (1973), quienes introdujeron una perspectiva sobre los sesgos heurísticos que ejecutan los humanos en un sin número de situaciones, se produjo un torrente de investigaciones en el campo de la psicología y las adicciones, y desde las cuales el estudio sobre la ludopatía no fue la excepción.

Las distorsiones cognitivas asociadas a las ganancias, potencian el desencadenamiento de la adicción, las evaluaciones sesgadas de los resultados en los juegos hacen que la persona adicta a las apuestas recuerde las ganancias de forma selectiva, es decir, sobrevaloran las positivas y tienden a olvidar las negativas, o a darles alguna explicación poco razonable (Ladouceur, 2004).

Los errores cognitivos remarcan las características de los ludópatas no solo como factor de riesgo, son también mantenedores de la conducta, convirtiéndose en parte fundamental y medular en este tipo de trastorno; autores como (Tobón, Cano \& Londoño, 2010) muestran cómo en la población ludópata predominan esquemas maladaptitvos tempranos de desconfianza, grandiosidad y pensamientos automáticos de filtraje y falacia de cambio.

Diferentes autores coinciden en afirmar que la forma como el individuo percibe, interpreta y valora los distintos elementos implicados en el juego y sus resultados, son los que marcan diferencia entre las personas jugadoras patológicas, las ocasionales, y las no jugadoras (Secades \& Villa citado en Estévez \& Calvete, 2007).

Aunque no es claro si estas cogniciones disfuncionales son previas o si surgen a raíz del juego mismo (Joukhador, Blaszczynski y Maccallum, 2004), lo evidente es que influyen de forma

E $\| 34$ 
perentoria y generan una incapacidad percibida para detener la conducta de juego (Jefferson \& Nicki, 2003; Mañoso et al., 2004; Ravlu \& Oei, 2004).

Desde comienzos de siglo se han realizado estudios para observar cómo la edad del jugador afecta el manejo de las emociones en el momento de tomar decisiones. Se sabe que hay cambios en la regulación emocional y la motivación en los adultos de mediana edad (30 a 60 años) y adultos mayores (60 o más años), (Carstensen, Fung, \& Charles, 2003). Estos últimos parecen ser más hábiles que los adultos más jóvenes de 18 a 30 años en la regulación emocional, y también han aumentado la complejidad emocional (Labouvie, 2005). Al observar estas características en momentos de tomar riesgos económicos autores como Word, Busemeyer y Stout (2002), concluyen que los adultos mayores tendrán mejor manejo de sus finanzas.

Por último, cabe destacar que los estudios en condiciones adictivas en la ludopatía se han enfocado en las relaciones entre abuso de sustancias y toma de decisiones. Fridberg, et al. (2010), han encontrado que factores como la ingesta de alcohol o las drogas cambian las percepciones emocionales haciendo más fácil la toma de riesgos en todo nivel.

\section{Clima familiar en jugadores}

La vida de un jugador patológico está rodeada de múltiples conflictos que comprometen de forma negativa sus relaciones familiares. Las continuas pérdidas de dinero requerido para los gastos fundamentales son el punto neurálgico de la problemática. Cuando la situación se agrava pasa a ser la familia la que es acosada por obligaciones financieras no pagadas por el ludópata.

Así mismo, es frecuente que la familia viva la exclusión social. Una forma es que los miembros mismos se marginen por la vergüenza que produce los problemas del adicto al juego o, también, puede existir pérdida de contacto con familiares y amigos porque son estos los que aíslan al jugador y su familia (Gaudia, 1987).

Pero los efectos no son solo relacionales. El constante estrés que genera el jugador a los suyos puede desembocar en problemas médicos diversos, sumados a las posibles implicaciones legales debido a las conductas delictivas del jugador patológico. 
Si el jugador es hombre, su pareja tiende a pensar inicialmente que está siendo engañada al no recibir explicaciones adecuadas y creíbles respecto a las ausencias recurrentes y falta de dinero. Esta situación puede conducir, entre otros, a rompimientos o a la depresión en la pareja del ludópata (Shaw, et al., 2007). Si quien juega es la mujer, existe una alta probabilidad que sea abandonada por su pareja, (Abbott, Cramer \& Sherrets, 1995). También pueden presentarse en la mujer cuadros depresivos, así como disfunciones sexuales que a la larga se convierten en fuente de problemas maritales, (Goldner, Rey \& Velázquez, 2013).

El ludópata cuando no está jugando tiende a buscar emociones fuertes que le proporcionen los niveles de adrenalina que encuentra al apostar. Esta necesidad de adrenalina lo lleva a desarrollar conductas sexuales adictivas que a su vez desencadenan conflictos al interior de la familia por infidelidades que pueden terminar en separaciones o desgastando el clima familiar existente. En un estudio realizado por Grant (2005) en el Hospital Providence en Rhode Island, Estados Unidos, demostró que de una muestra de 225 adictos al juego, 44 de ellos, es decir el $19.6 \%$ cumplen con los criterios para establecer presencia de conductas sexuales adictivas.

El Consejo Nacional para la Investigación Americana reportó para el año 1999 que del 25 al 50 $\%$ de hijos de jugadores patológicos eran maltratados físicamente o presentaban denuncias por abandono o negligencia en su rol paterno. El informe también revela que en las comunidades donde se establecieron nuevos casinos, aumentó la violencia doméstica que en muchas ocasiones involucró a niños; todo esto debido a que los progenitores descuidaban a sus hijos por frecuentar los sitios de juego (Domínguez, 2009).

Cuando los niños hijos de ludópata alcanzan la edad adulta, es probable que aparezca en ellos dicho problema. Al menos el $24 \%$ de los adultos con problemas de juego crecieron en familias donde hubo la misma situación (National Opinion Center, 1999).

\section{Apuestas por internet}

En la actualidad, el internet ha abierto nuevas modalidades de juegos de azar que solamente han sido estudiados en los últimos años; casinos virtuales y diferentes tipos de juegos de azar electrónicos, con apuestas de dinero real, donde los jugadores pueden ingresar fondos desde

$\boldsymbol{\epsilon} \mid 36$ 
sus tarjetas de crédito o cuentas bancarias. Los ingresos obtenidos a nivel mundial hasta el año 2000 por parte de las apuestas en casinos de Internet ascendió a 2.2 billones de dólares (Hammer, 2001). Sin embargo, las pérdidas de cartera vencida en las entidades bancarias fue manifiesta en los años venideros, dado que los ahorradores perdían grandes sumas de dinero de sus tarjetas de crédito $y$, finalmente, estos créditos no eran cancelados a la entidad financiera.

Solo hasta el año 2003, las entidades bancarias en Estados Unidos reportaron disminución de pérdidas en tarjetas de crédito de 5 billones a 1,2 billones gracias a la prohibición del acceso ilimitado de fondos para sitios Web de apuestas (Depaula, 2002).

El afán de producir más ganancias ha llevado a diseñar diversas estrategias para la búsqueda de nuevos jugadores, es por esto que los sitios de apuestas por internet cada vez son más prolíficos y utilizan medios persuasivos como bonos de juego gratis que invitan al público en general a participar en dichas páginas.

Un estudio realizado con personas que asistían a centros clínicos (Griffiths, 2006) por diferentes problemáticas reveló que, el 6,9 \% de las personas reportaron haber jugado dinero por internet y que el 65,9\% presentaban características para ser diagnosticadas con problemas de juego, y jugaban frecuentemente por internet; estas cifras son similares en estudios de prevalencia en adolescentes que asisten al colegio (Weinstock, 2007) y en diferentes países (Ladd, et al., 2002).

Si se tiene en cuenta que los niños y jóvenes de menor edad son las personas más cercanas a los medios electrónicos, y que la edad temprana en la iniciación del juego es un factor determinante en el futuro del ludópata. Es prioritario, entonces, analizar y estudiar con detenimiento sitios de acceso fácil al juego para buscar estrategias preventivas de posibles adictos al juego. Solo hasta los últimos años (Pérez, 2012) se ha visto el interés por diseñar y aplicar métodos para la regulación de las apuestas en casinos virtuales. De esta forma, se busca reducir el impacto de dichos juegos en las finanzas de los jugadores y minimizar los riesgos de adicción en los mismos.

En el cuestionario aplicado para este estudio se adicionó una pregunta referente al juego de apuestas por internet con el fin de observar el número de jugadores que realizaron este tipo de actividades dentro de los grupos estudiados. 


\section{Método}

Se llevó a cabo un estudio exploratorio a partir de la aplicación del cuestionario South Oaks Gambling Score (SOGS), para identificar posibles jugadores patológicos; se dividieron los participantes en cuatro grupos según la edad y resultado en el SOGS; se aplicaron escalas de sesgos cognitivos y clima familiar para los diferentes grupos.

\section{Participantes}

La muestra para este estudio está compuesta por 60 personas entre 18 y 35 años y 60 personas entre 36 y 60 años, todos residentes en la ciudad de Bogotá y con estrato socioeconómico entre el 1 y 5 . Los participantes fueron divididos en cuatro grupos de 30 personas teniendo en cuenta los baremos de calificación del South Oaks Gambling Score ( SOGS) que es el instrumento más fiable para la detección de juego patológico (Echeburúa \& Fernández 2002) en su versión validada en castellano (Lesieur \& Blume, 1993). Dicho formulario propone como jugadores patológicos a participantes con puntuaciones mayores a 4.

Para mayor facilidad, los grupos 1 y 3 serán catalogados como grupos de jugadores por tener puntuaciones de 5 o más, y los grupos 2 y 4 como no jugadores por tener puntuaciones de 3 o menos. Los cuestionarios con puntajes de 4 fueron omitidos ya que dicha puntuación marca el límite para determinar un problema de juego, solo se tomaron en cuenta los cuestionarios que mostraron puntuaciones superiores o inferiores a este puntaje, esto con el fin de distinguir de mejor manera los grupos y realizar comparaciones efectivas entre grupos.

Tabla 1 / Organización de Grupos

\begin{tabular}{cccccc}
\hline \hline GRUPO & CATEGORÍA & EDAD & $\begin{array}{c}\text { MEDIA DE } \\
\text { EDAD }\end{array}$ & MUJERES & HOMBRES \\
\hline \hline 1 & Jugadores & 18 a 35 & 22,23 & 3 & 27 \\
\hline 2 & $\begin{array}{c}\text { No } \\
\text { Jugadores }\end{array}$ & 18 a 35 & 22,13 & 9 & 21 \\
\hline 3 & Jugadores & 36 a 60 & 45,43 & 8 & 22 \\
\hline 4 & $\begin{array}{c}\text { No } \\
\text { Jugadores }\end{array}$ & 36 a 60 & 44,86 & 5 & 25 \\
\hline
\end{tabular}

Fuente: Tabla de elaboración propia.

$\boldsymbol{\epsilon} \| 38$ 


\section{Instrumentos}

Se utilizó el South Oaks Gambling Screen (versión en castellano), abreviadamente SOGS, de Lesieur y Blume (1987). Este instrumento consta de 20 ítems y se adapta a los criterios del DSMIV-TR (APA, 2000). Los ítems evalúan las relaciones, conducta de juego y fuentes de obtención del dinero para jugar o para pagar las deudas, así como las emociones implicadas. En la versión española, una puntuación igual o superior a 5 indica la presencia del juego patológico, puntuaciones de 2 o 3 se catalogan como personas con posibles problemas con el juego. Además, la puntuación obtenida en este cuestionario constituye un reflejo de la gravedad de la adicción al juego ${ }^{3}$. Para puntuar al sujeto en el SOGS, se tienen en cuenta las respuestas a las preguntas 6 a 17. Las preguntas 5 y 18 forman parte del instrumento pero no se tienen en cuenta para el cálculo del puntaje en la prueba SOGS, sino para obtener información adicional sobre sus patrones de juego y de gravedad del problema.

Para el segundo bloque se utilizó el Inventario de pensamientos sobre el juego (Echeburúa et al. 1994), adaptado por Ruiz y García (2008), que consiste en un listado de 11 ítems binarios cuya respuesta puede ser sí o no, y están dirigidos a detectar los pensamientos irracionales del jugador patológico, este instrumento permite detectar los sesgos cognitivos presentes en relación a las conductas de juego.

Por su parte, el Inventario sobre Clima Familiar fue utilizado para estudios sobre dinámicas familiares realizados por el Instituto Colombiano de Bienestar Familiar (Ruiz ob cit., 2008). Este instrumento consta de dos bloques de preguntas que buscan evaluar las relaciones familiares y las actividades en familia, con el objetivo de determinar el grado de cohesión y apoyo social dentro del núcleo familiar del participante. La puntuación obtenida se da a través de una escala tipo Likert así: (a) Casi siempre es igual = 4 puntos, (b) Con frecuencia $=3$ puntos, (c) Algunas Veces $=2$ puntos, (d) Muy rara vez $=1$ punto, (e) Nunca $=0$ puntos. El puntaje máximo obtenido es de 40 y supone un excelente clima familiar; puntajes por debajo de 30 manifestarían algunas dificultades al interior de la familia o ausencia de la misma para realizar actividades sociales.

\footnotetext{
${ }^{3}$ Un análisis más detallado de las propiedades psicométricas del SOGS puede encontrarse en Echeburúa et al. (1994).
} 


\section{Procedimiento}

Los participantes fueron entrevistados en lugares habituales de apuestas como casinos y sitios de juego de la ciudad de Bogotá y en diferentes universidades de la ciudad, además, fueron informados del objeto del estudio y accedieron a participar de manera voluntaria firmando documento de consentimiento.

\section{Análisis Estadístico}

El análisis estadístico se realizó por medio de los programas SPSS 11.0 versión en castellano. También se utilizó el Sistema Portátil para el Análisis de Datos Cualitativos -SPADT- (por sus siglas en francés), versión 1.5 y Excel para Windows; en el inventario SOGS se distinguió de forma separada a las personas que marcaron puntajes de 2 o 3 y las personas con puntajes de 5 o más. Se dividió la variable de clima familiar en Pésimo, Malo, Regular y Bueno para observar de forma más ilustrativa las relaciones entre las diferentes variables analizadas.

Para establecer las características de la muestra se utilizó el procedimiento de KolmogorovSmirnov, y luego de determinar que hay una distribución normal en los datos de la muestra se realiza una comparación de las medias obtenidas en las diferentes escalas analizadas en los cuatro grupos mencionados anteriormente, después, se realizó unas correlaciones de Pearson entre los grupos de jugadores jóvenes y adultos para observar su relación con los puntajes en el formulario del SOGS.

\section{Resultados}

Los resultados obtenidos parten de las diferencias de medias y de las correlaciones realizadas entre los grupos dependiendo de los puntajes en adicción al juego, sesgos cognitivos y clima familiar. En las tablas 2, 3 y 4 se presenta las medias de cada escala, y posteriormente, en la tabla 5 , se pueden observar las diferencias entre los grupos. 
Tabla 2 | Anova puntajes cuestionario sogs

\begin{tabular}{cccccrc}
\hline \hline & N & MEDIA & $\begin{array}{c}\text { DESVIACIÓN } \\
\text { TíPICA }\end{array}$ & $\begin{array}{c}\text { ERROR } \\
\text { TíPICO }\end{array}$ & $\begin{array}{c}\text { INTERVALO DE } \\
\text { CONFIANZA PARA LA } \\
\text { MEDIA AL 95\% }\end{array}$ \\
\cline { 2 - 7 } GRUPO & & & & & LíMITE & $\begin{array}{r}\text { LíMITE } \\
\text { INFERIOR }\end{array}$ \\
\hline \hline 1 & 30 & 7,50 & 2,080 &, 380 & 6,72 & 8,28 \\
\hline 2 & 30 & 1,13 & 1,332 &, 243 & 0,64 & 1,63 \\
\hline 3 & 30 & 7,70 & 2,466 &, 450 & 6,78 & 8,62 \\
\hline 4 & 30 & 1,10 & 1,348 &, 246 & 0,60 & 1,60 \\
\hline Total & 120 & 4,36 & 3,743 &, 342 & 3,68 & 5,03 \\
\hline
\end{tabular}

Fuente: Tabla de elaboración propia.

Tabla 3 / ANOVA Puntajes Sesgos Cognitivos

\begin{tabular}{ccccccc}
\hline \hline & MEDIA & $\begin{array}{c}\text { DESVIACIÓN } \\
\text { TíPICA }\end{array}$ & $\begin{array}{c}\text { ERROR } \\
\text { TíPICO }\end{array}$ & \multicolumn{2}{c}{$\begin{array}{c}\text { INTERVALO DE } \\
\text { CONFIANZA PARA LA } \\
\text { MEDIA AL 95\% }\end{array}$} \\
\cline { 2 - 7 } GRUPO & & & & & $\begin{array}{c}\text { LíMITE } \\
\text { INFERIOR }\end{array}$ & $\begin{array}{c}\text { LÍMITE } \\
\text { SUPERIOR }\end{array}$ \\
\hline \hline 1 & 30 & 6,17 & 2,052 & 0,375 & 5,40 & 6,93 \\
\hline 2 & 30 & 2,70 & 1,535 & 0,280 & 2,13 & 3,27 \\
\hline 3 & 30 & 4,83 & 1,783 & 0,325 & 4,17 & 5,50 \\
\hline 4 & 30 & 2,00 & 1,800 & 0,329 & 1,33 & 2,67 \\
\hline Total & 120 & 3,93 & 2,440 & 0,223 & 3,48 & 4,37 \\
\hline
\end{tabular}

Fuente: Tabla de elaboración propia. 
Tabla 4 / ANOVA Puntajes Clima Familiar

\begin{tabular}{|c|c|c|c|c|c|c|}
\hline \multirow[b]{2}{*}{ GRUPO } & \multirow[t]{2}{*}{$\mathbf{N}$} & \multirow[t]{2}{*}{ MEDIA } & \multirow[t]{2}{*}{$\begin{array}{l}\text { DESVIACIÓN } \\
\text { TÍPICA }\end{array}$} & \multirow[t]{2}{*}{$\begin{array}{l}\text { ERROR } \\
\text { Tí́PICO }\end{array}$} & \multicolumn{2}{|c|}{$\begin{array}{l}\text { INTERVALO DE } \\
\text { CONFIANZA PARA LA } \\
\text { MEDIA AL } 95 \%\end{array}$} \\
\hline & & & & & $\begin{array}{r}\text { LÍMITE } \\
\text { INFERIOR }\end{array}$ & $\begin{array}{r}\text { LÍMITE } \\
\text { SUPERIOR } \\
\end{array}$ \\
\hline 1 & 30 & 23,17 & 7,773 & 1,419 & 20,26 & 26,07 \\
\hline 2 & 30 & 24,23 & 7,482 & 1,366 & 21,44 & 27,03 \\
\hline 3 & 30 & 20,23 & 8,787 & 1,604 & 16,95 & 23,51 \\
\hline 4 & 30 & 29,80 & 5,738 & 1,048 & 27,66 & 31,94 \\
\hline Total & 120 & 24,36 & 8,205 & 0,749 & 22,88 & 25,84 \\
\hline
\end{tabular}

Fuente: Tabla de elaboración propia.

Tabla 5 / Comparaciones Bonferroni entre grupos

\section{Comparaciones múltiples}

\begin{tabular}{lllc}
\hline $\begin{array}{c}\text { VARIABLE } \\
\text { DEPENDIENTE }\end{array}$ & \multicolumn{1}{c}{ GRUPO } & \multicolumn{1}{c}{ GRUPO } & $\begin{array}{c}\text { DIFERENCIA DE } \\
\text { MEDIAS }(*)\end{array}$ \\
\hline \hline P. SOGS & Jóvenes jugadores & jóvenes no jugadores & $6,37(*)$ \\
& & adultos jugadores &,- 20 \\
& Jóvenes no jugadores & adultos no jugadores & $6,40(*)$ \\
& jóvenes jugadores & $-6,37(*)$ \\
& Adultos jugadores & adultos jugadores & $-6,57(*)$ \\
& adultos no jugadores &, 03 \\
& jóvenes jugadores &, 20 \\
& jóvenes no jugadores & $6,57(*)$ \\
& Adultos no jugadores & adultos no jugadores & $6,60\left(^{*}\right)$ \\
& & jóvenes jugadores & $-6,40(*)$ \\
& jóvenes no jugadores &,- 03 \\
& & adultos jugadores & $-6,60(*)$ \\
\hline
\end{tabular}

$\boldsymbol{\epsilon} \| 42$ 


\begin{tabular}{|c|c|c|c|}
\hline $\begin{array}{c}\text { VARIABLE } \\
\text { DEPENDIENTE }\end{array}$ & GRUPO & GRUPO & $\begin{array}{l}\text { DIFERENCIA DE } \\
\text { MEDIAS (*) }\end{array}$ \\
\hline \multirow[t]{11}{*}{ P. FAMILIA } & \multirow[t]{3}{*}{ Jóvenes jugadores } & jóvenes no jugadores & $-1,07$ \\
\hline & & adultos jugadores & 2,93 \\
\hline & & adultos no jugadores & $-6,63\left(^{*}\right)$ \\
\hline & \multirow[t]{2}{*}{ Jóvenes no jugadores } & $\begin{array}{l}\text { jóvenes jugadores } \\
\text { adultos jugadores }\end{array}$ & $\begin{array}{l}1,07 \\
4,00\end{array}$ \\
\hline & & adultos no jugadores & $-5,57\left(^{*}\right)$ \\
\hline & \multirow[t]{3}{*}{ Adultos jugadores } & jóvenes jugadores & $-2,93$ \\
\hline & & jóvenes no jugadores & $-4,00$ \\
\hline & & adultos no jugadores & $-9,57\left(^{*}\right)$ \\
\hline & \multirow[t]{3}{*}{ Adultos no jugadores } & jóvenes jugadores & $6,63(*)$ \\
\hline & & jóvenes no jugadores & $5,57(*)$ \\
\hline & & adultos jugadores & $9,57(*)$ \\
\hline \multirow[t]{11}{*}{ P. SESGOS } & \multirow[t]{3}{*}{ Jóvenes jugadores } & jóvenes no jugadores & $3,47(*)$ \\
\hline & & adultos jugadores & $1,33(*)$ \\
\hline & & adultos no jugadores & $4,17(*)$ \\
\hline & \multirow[t]{3}{*}{ Jóvenes no jugadores } & jóvenes jugadores & $-3,47\left(^{*}\right)$ \\
\hline & & adultos jugadores & $-2,13(*)$ \\
\hline & & adultos no jugadores & ,70 \\
\hline & \multirow[t]{2}{*}{ Adultos jugadores } & jóvenes jugadores & $-1,33\left(^{*}\right)$ \\
\hline & & $\begin{array}{l}\text { jóvenes no jugadores } \\
\text { adultos no jugadores }\end{array}$ & $\begin{array}{l}2,13(*) \\
2,83(*)\end{array}$ \\
\hline & \multirow[t]{3}{*}{ Adultos no jugadores } & jóvenes jugadores & $-4,17(*)$ \\
\hline & & jóvenes no jugadores &,- 70 \\
\hline & & adultos jugadores & $-2,83\left(^{*}\right)$ \\
\hline
\end{tabular}

* La diferencia entre las medias es significativa al nivel .05.

Como se observa en la tabla 5, no existe diferencia significativa en los puntajes del SOGS entre el grupo de jugadores jóvenes y jugadores adultos, ni entre los grupos de no jugadores jóvenes y no jugadores adultos. Las diferencias se encuentran entre los grupos de jugadores y no jugadores independiente de la edad con $(F=120.16, p<0.05)$. 
En cuanto al puntaje de la escala de sesgos cognitivos, se encontró una diferencia significativa $(F=34.053, p<0.05)$, entre los grupos de jugadores y no jugadores independiente de la edad y se evidenció una diferencia significativa entre los grupos de jugadores menores de 35 años con los jugadores mayores de 35 años.

Respecto al puntaje en la escala de clima familiar se halló una diferencia significativa entre los grupos de jugadores y no jugadores independiente de la edad, También entre el grupo de jóvenes no jugadores y adultos no jugadores se descubrió diferencia significativa $(F=8.48, p<0.05)$. A continuación se presenta en la tabla 6 la síntesis de las medias obtenidas de cada grupo en las diferentes escalas que se analizaron.

Tabla 6 / Síntesis de Medias obtenidas por cada grupo en las escalas analizadas.

\begin{tabular}{|c|c|c|c|c|}
\hline & GRUPO & PUNTAJE SOGS & PUNTAJE SESGOS & $\begin{array}{l}\text { PUNTAJE } \\
\text { FAMILIA } \\
\end{array}$ \\
\hline 1. & Jóvenes Jugadores & 7,50 & 6,17 & 23,17 \\
\hline 2. & Jóvenes No jugador & 1,13 & 2,70 & 24,23 \\
\hline 3. & Adultos Jugadores & 7,70 & 4,83 & 20,23 \\
\hline & Adultos No Jugador & 1,10 & 2,00 & 29,80 \\
\hline
\end{tabular}

Fuente: Tabla de elaboración propia.

Al realizar comparaciones entre los cuatro grupos buscando relaciones con respecto a las puntuaciones en las escalas de sesgos cognitivos y clima familiar con una significancia de 0.05 , se percibió que el puntaje en la escala de sesgos cognitivos se relaciona de manera positiva moderada con el puntaje obtenido en el SOGS en el grupo de jóvenes jugadores ( $r=4.35$ ) y el grupo de Adultos jugadores, $(r=4.17$. 
En lo referente a la relación entre el puntaje obtenido en el SOGS y el clima familiar, el único grupo que mostró una relación negativa moderada fue el de Adultos no Jugadores $(r=4.1)$ con un nivel de significancia de 0.05

En lo referente a la pregunta que se realizó sobre el juego por Internet, se puede contrastar porcentajes altos en los grupos de Jugadores como se muestra a continuación en la tabla 7.

Tabla 7 / Porcentajes de personas que realizaron apuestas por Internet en cada grupo

\begin{tabular}{cc}
\hline \hline GRUPO & PORCENTAJE \\
\hline \hline 1 & $51 \%$ \\
2 & $10 \%$ \\
3 & $26,6 \%$ \\
4 & $0,0 \%$ \\
\hline
\end{tabular}

Fuente: Tabla de elaboración propia.

Los grupos de jóvenes jugadores fueron los que más afirmaron haber hecho apuestas por Internet o en casinos virtuales con un $51 \%$, seguidos de los adultos jugadores con un $26.6 \%$. Cabe destacar que dentro del grupo de jóvenes no jugadores también un $10 \%$ de ellos respondieron afirmativamente a la pregunta. En el único grupo que nadie aseguró haber realizado este tipo de actividades fue en el de adultos no jugadores.

A continuación, se muestra en la tabla 8 las variables que más se relacionaron en cada uno de los grupos con sus porcentajes, resultado encontrado por medio del sistema de descripción de variables nominales del software SPAD. 
Tabla 8 / Variables más relacionadas en cada grupo con su respectivo porcentaje

\begin{tabular}{|c|c|c|c|c|}
\hline GRUPO & VARIABLE & $\begin{array}{c}\text { PORCENTAJE } \\
\text { TOTAL } \\
\text { MUESTRA }\end{array}$ & $\begin{array}{l}\text { PORCENTAJE EN } \\
\text { EL GRUPO }\end{array}$ & VALOR TEST \\
\hline 1 & $\begin{array}{l}\text { Muchos sesgos } \\
\text { Si jugo por } \\
\text { Internet, } \\
\text { Soltero }\end{array}$ & $\begin{array}{l}14,17 \% \\
22,50 \% \\
56,67 \%\end{array}$ & $\begin{array}{c}43,33 \% \\
59,26 \% \\
80 \%\end{array}$ & $\begin{array}{l}4,64, p=.000 \\
4,64, p=.000 \\
2,84, p=.002\end{array}$ \\
\hline 2 & $\begin{array}{l}\text { Pocos sesgos } \\
\text { Soltero }\end{array}$ & $\begin{array}{l}37,50 \% \\
22,50 \%\end{array}$ & $\begin{array}{l}66,67 \% \\
96,67 \%\end{array}$ & $\begin{array}{l}3,55, p=.000 \\
5,35, p=.000\end{array}$ \\
\hline 3 & $\begin{array}{c}\text { Bastantes } \\
\text { Sesgos estrato } 2 \\
\text { Pésimo clima F. } \\
\text { casado }\end{array}$ & $\begin{array}{c}30 \% \\
29,17 \% \\
25 \% \\
24,17 \%\end{array}$ & $\begin{array}{c}60 \% \\
5,33 \% \\
46,33 \% \\
50 \%\end{array}$ & $\begin{array}{l}3,80, p=.000 \\
3,04, p=.001 \\
2,37, p=.009 \\
3,42, p=.000\end{array}$ \\
\hline 4 & $\begin{array}{l}\text { No juego por } \\
\text { Internet Muy } \\
\text { Pocos Sesgos } \\
\text { Buen clima F. } \\
\text { Casado }\end{array}$ & $\begin{array}{l}77,50 \% \\
18,33 \% \\
23,33 \% \\
24,17 \%\end{array}$ & $\begin{array}{c}100 \% \\
40 \% \\
43,33 \% \\
43,33\end{array}$ & $\begin{array}{l}3,65, p=.000 \\
3,11, p=.001 \\
2,65, p=.004 \\
2,51, p=.006\end{array}$ \\
\hline
\end{tabular}

Fuente: Tabla de elaboración propia.

En la tabla anterior se pueden ver algunas relaciones de porcentajes que dan información adicional sobre cada uno de los grupos, por ejemplo, en el grupo 1 un $43.33 \%$ de los participantes se caracterizó por tener muchos sesgos, de igual forma en el grupo 3, un $60 \%$ de los participantes se caracterizaron por tener bastantes sesgos, además, un $46.33 \%$ de los participantes de este grupo se caracterizaron por tener un pésimo clima familiar. Estos resultados son opuestos en los grupos 2 y 4 , donde predomina los porcentajes de participantes con pocos sesgos, $66.67 \%$ en el grupo 2 , y muy pocos sesgos, $40 \%$, de los participantes del grupo 4 , además, en este grupo el porcentaje de personas con buen clima familiar fue del $43.3 \%$.

$\boldsymbol{E} \mid 46$ 


\section{Discusión}

Al analizar los resultados se observan diferencias esperadas entre los grupos de jugadores y no jugadores en lo referente a las escalas en el SOGS independiente de la edad, dichos hallazgos corroboran los múltiples estudios (Ferris \& Wynne, 2001; Vacca, 2005), en los cuales los ludópatas han demostrado tener siempre puntuaciones altas con el instrumento utilizado. En la población Bogotana se cumplió de la misma forma dichas diferencias, pues muestra la eficacia del cuestionario del SOGS para hacer este tipo de investigaciones y la marcadas diferencias que existen en comportamientos y emociones de personas que acostumbran a apostar y las que no.

En cuanto a la escala de sesgos cognitivos, se dan diferencias entre los grupos de jugadores y no jugadores sin importar la edad, esto es compatible con los estudios de Echeburua, 2002; Chóliz, 2006; Jefferson y Nicki, 2003, los cuales afirman que los sujetos ludópatas presentan más errores cognitivos y una menor capacidad para interpretar de manera razonable las situaciones. Lo interesante para destacar es la diferencia que se encontró entre los puntajes de dicha escala en los grupos de jugadores menores de 35 años y los mayores de 35 años, dicha discrepancia puede estar cercana a los hallazgos de Carstensen, Fung, y Charles (2003), los cuales demostraron que existen cambios en la regulación emocional dependiendo de la edad. Estos autores suponen que hay una mejor regulación emocional después de los 30 años y que hay un cambio en la motivación, lo que supondría diferentes formas de afrontar las situaciones de riesgo. En el juego, específicamente, esta diferencia se relacionaría con los resultados de los estudios de Word y otros en 2005, en los cuales las personas de mayor edad mostraron un mejor rendimiento en tareas de decisiones económicas y mostraron ser más consistentes en el manejo de sus finanzas que las personas más jóvenes puesto que asumían menores riesgos sobre su economía.

A pesar que los resultados muestran grados de adicción igual en jugadores jóvenes y adultos, las emociones implicadas en el juego y la forma de asumir los riesgos financieros podrían explicar por qué la mayor parte de los ludópatas empiezan a jugar de forma descontrolada a edades tempranas, y además se ratificaría la edad como un factor de riesgo para la adicción al juego (Shaffer \& Hall, 2001). 
En lo referente a la escala de clima familiar, se encontraron puntajes más bajos en aquellos que se situaron como jugadores patológicos, esto concuerda con autores como Gaudia (1987) y Becoña (2007), quienes afirman que la ludopatía trae un deterioro paulatino al interior de la familia. Asimismo, autores como Shaw y et.al (2007) y Abbott, Cramer, Sherrets, (1995) afirman que la ludopatía trae consecuencias de exclusión y aislamiento al interior de la familia, esto podría analizarse en estudios posteriores donde fueran entrevistados familiares cercanos de personas adictas al juego y determinar de qué manera se cumplen estas afirmaciones en nuestro país.

Un dato interesante es la relación entre el estado civil divorciado y las variables características del jugador patológico, lo que deja interrogantes acerca de si las condiciones de estado civil podrían relacionarse con el hecho de que una persona se acerque más a los juegos de azar, la inestabilidad al interior de la familia o de la pareja podría suponer búsqueda de emociones fuertes o despilfarro de dinero en algún momento.

Es posible que existen relaciones directas entre el nivel de estudios y los puntajes en la escala de sesgos cognitivos, esto hace suponer que la capacidad para tomar decisiones no segadas al menos en lo referente al azar podría relacionarse, no solo con la maduración cognitiva referida a la edad, si no también con las herramientas intelectuales de las que haga uso la persona.

Aunque la literatura reporta que hay una relación directa entre el sexo masculino y el ser ludópata, (Bahamon, 2006). Los resultados presentados en este trabajo no parecen mostrar esa relación, sin embago, el tamaño de la muestra y el número de participantes del sexo femenino no es suficiente para corroborar dicha afirmación.

Uno de los datos más interesantes del presente análisis son los porcentajes de personas que acostumbran a jugar dinero en casinos virtuales. Más de la mitad de los jóvenes que puntuaron alto en el SOGS, afirmaron haber realizado este tipo de apuestas. También, un $26 \%$ de los jugadores mayores de 35 dijeron haber realizado esta misma clase de actividades. Dentro del grupo de no jugadores un $10 \%$ de la población menor de 35 años afirmó haber realizado estos juegos.

\section{Conclusiones}

La manera en la cual el sujeto se ancla al juego y la forma como las emociones se convierte en mantenedores de una conducta adictiva, es un punto crucial para tener en cuenta, sobre todo 
en la población joven puesto que puede generar múltiples problemáticas, no solo con el juego, sino también en diferentes conductas adictivas perjudiciales para el individuo.

La ingesta de alcohol descontrolada, conductas delincuenciales, exposición a situaciones de riesgo podrían estar relacionadas con sesgos cognitivos que influyen en la toma de decisiones, por lo cual debe ser un tema de gran atención en el futuro.

Hay que destacar que en este estudio se encontró menores puntajes en el clima familiar en el grupo de adultos no jugadores, comparados con el de los jóvenes no jugadores; esto pone de presente la posibilidad que el debilitamiento del clima familiar, al menos en nuestra cultura, esté relacionado con la edad del sujeto, sin importar si tiene algún tipo de adicción o no.

Los resultados que refieren al juego por internet muestran la creciente incursión de este tipo de apuestas en lo que respecta a las actividades de ocio, los casinos en línea han pasado a constituirse como un fenómeno de gran escala a nivel mundial y nuestro país parece no ser ajeno a dicha dinámica. Las problemáticas asociadas al juego adictivo en estos sitios están en el marco de la ludopatía; el fácil acceso a estos sitios, las nuevas tendencias sociales respecto a internet, el acercamiento de las personas más jóvenes a los medios electrónicos pueden acarrear problemáticas familiares y sociales que deben ser estudiadas con más detenimiento para poder generar planes de prevención al respecto.

\section{Referencias}

Abbott, D., Cramer, S. \& Sherrets, S. (1995). Pathological Gambling and the Family: Practice Implications. Families in Society, 76(4), 213-219. Recuperado de http://psycnet.apa.org/psycinfo/1995-37189-001

Bahamon, M. (2006). Juego patológico: revisión de tema. Revista Colombiana de Psiquiatría, 35(3), 380-399. Recuperado de http://www.scielo.org.co/pdf/rcp/v35n3/v35n3a07

Becoña, E. (2007). Bases psicológicas de la prevención del consumo de drogas. Papeles del Psicólogo, 28(1), 1120. Recuperado de http://www.psiquiatria.com/psicologia/bases-psicologicas-de-la-prevencion-delconsumo-de-drogas/

Busemeyer, J., \& Stout, J. (2002). A contribution of cognitive decision models to clinical assessment: Decomposing performance on the Bechara gambling task. Psychological Assessment. 14(3), 253-262. Recuperado de http://www.ncbi.nlm.nih.gov/pubmed/12214432 
Carstensen, L., Fung, H., \& Charles, S. (2003). Socioemotional selectivity theory and regulation of emotion in the second half of life. Journal Motivation and Emotion, 27(2), 103-123. doi: 110.1023/A:1024569803230.

Chóliz, M. (2006). Adicción al juego: sesgos y heurísticos implicados en los juegos de azar. Revista española de Drogodependencias, 31(2), 173-184. Recuperado de http://www.aesed.com/descargas/revistas/ v31n2_3.pdf

DANE, (2008). Encuesta de Calidad de Vida 2008 presentación de resultados.

Depaula, M. (2002). On-line gambling craps out with credit card billers. US Banker, 112(23), 23. Recuperado de http://connection.ebscohost.com/c/articles/7118125/on-line-gaming-craps-out-credit-card-billers

Domínguez, A. (2009). Epidemiología y factores implicados en el juego patológico. Apuntes de Psicología, 27(1), 3-20. Recuperado de http://www.jogoremoto.com/docs/extra/xiR7At.pdf

Echeburúa, R. \& Fernández , M. (2002). Juego patológico y adicciones sin drogas: Tratamiento. Proyecto Hombre: Revista Trimestral de la Asociación Proyecto Hombre, 44, 13-16.

Echeburúa, E., Báez, C., Fernández-Montalvo, J. \& Páez, D. (1994). Cuestionario de juego patológico de South Oaks (SOGS): validación española. Análisis y Modificación de Conducta, 20(74), 769-791. Recuperado de http://www.researchgate.net/publication/234736399_Cuestionario_de_Juego_Patolgico_de_South_ Oaks_(SOGS)_validacin_espaola

Esteves, A. \& Calvete, E. (2007). Esquemas cognitivos en personas con conducta de juego patológico y su relación con experiencias de crianza. Clínica y Salud, 18(1), 23-43. Recuperado de http://scielo.isciii.es/pdf/ clinsa/v18n1/v18n1a03.pdf

Ferris, J. \& Wynne, H. (2001). The Canadian Problem Gambling Index: Final Report. Recuperado de: http://www. ccsa.ca/Eng/Priorities/Gambling/CPGI/Pages/default.aspx. FinalReport

Fernández, J. (1997). Manual práctico de juego patológico ayuda para el paciente y guía para el terapeuta. Madrid: Editorial Pirámide.

Fernández, J., Landa, N., López, J. (2005). Prevalencia del juego patológico en el alcoholismo: un estudio exploratorio. Revista de Psicopatología y Psicología Clínica, 10(2), 125-134. Recuperado de http://aepcp.net/ arc/03.2005(2).Fernandez-Montalvoetal.pdf

E $\mid 50$ 
Fridberg, D., Queller, S., Ahn, W., Kim, W., Bishara, A., Busemeyer, J., et al. (2010). Cognitive mechanisms underlying risky decision-making in chronic cannabis users. Journal of Mathematical Psychology. 54(1), 28-38. Recuperado de http://www.sciencedirect.com/science/article/pii/S0022249609001205

Gaudia, R. (1987). Effects of Compulsive Gambling on the Family. Social Work, 32(3), 254-256. Recuperado de http://www.jstor.org/stable/23715190?seq=1\#page_scan_tab_contents

Goldner, J., Rey, L., Velázquez, A. (2013). Ludopatía en mujeres, un estudio de caso. Alternativas en psicología. 17(28), 33-40. Recuperado de http://alternativas.me/attachments/article/30/3.\%20Ludopatia\%20 en\%20mujeres-\%20un\%20estudio\%20de\%20caso.pdf

Grant, E. (2005). Compulsive Sexual Behavior and Pathological Gambling. Sexual Addiction \& Compulsivity: The Journal of Treatment \& Prevention, 12(2-3), 235-244. Recuperado de http://www.tandfonline.com/ doi/abs/10.1080/10720160500203856?journalCode=usac20\#.VeYVhSV_Oko

Griffiths, M. (2006). Internet gambling: an emerging concern in family practice medicine Family practice 23(4), 421-426.

Hammer, R. (2001) Does Internet Gambling Strengthen the U.S. Economy? Don't Bet On It. Federal Communications Law Journal, 54(1). Recuperado de http://www.repository.law.indiana.edu/fclj/vol54/iss1/6/ .

Jefferson, S. \& Nicki, R. (2003). A new instrument to measure cognitive distortions in video lottery terminal users: The Informational Biases Scale (IBS). Journal of Gambling Studies, 19(4), 387 - 403. Recuperado de http://www.ncbi.nlm.nih.gov/pubmed/14634299

Joukhador, J., Blaszczynski, A. \& Maccallum, F. (2004). Superstitious beliefs in gambling among problem and non-problem gamblers: Preliminary data. Journal of Gambling Studies, 20(2), 171-180. Recuperado de http://www.ncbi.nlm.nih.gov/pubmed/15060332

Kabneman, D., \& Tversky, A. (1973). On the psychology of prediction. Psycliological Review, 80(4), 237-251. Recuperado de http://lab4.psico.unimib.it/nettuno/forum/free_download/on_the_psychology_of_prediction_190.pdf

Labouvie, G. (2005). Emerging structures of adult thought. En: J. J. Arnett \& J. L. Tanner (Eds.), Emerging adults in America: Coming of age in the 21st century, pp. 59-84. Washington, DC, US: American Psychological Association. 
Ladd, George T. \& Petry, N. (2002). Disordered gambling among university-based medical and dental patients: A focus on Internet gambling. Psychology of Addictive Behaviors, 16(1), 76-69. Recuperado de https:// www.apa.org/pubs/journals/releases/adb-16176.pdf

Ladouceur, R., Mayrant, M. \& Tourigny, Y. (1987). The risk Taking Behavior in gamblers and non-gamblers during prolonged exposure. Journal Gambling Behavior, 3(2), 115-122. Recuperado de http://link.springer. com/article/10.1007\%2FBF01043450\#page-1

Laplante, D., \& Shaffer, H. (2007). Understanding the influence of gambling opportunities: Expanding exposure models to include adaptation. American Journal of Orthopsychiatry, 4(77), 616-623. Recuperado de http://www.ncbi.nlm.nih.gov/pubmed/18194041

Lesieur, H. (1993). Prevalencia, características y tratamiento de los jugadores patológicos en Estados Unidos. Psicología Conductual, 1(3), 389-407. Recuperado de http://www.funveca.org/revista/PDFespanol/1993/ num1/Prevalencia.pdf

Lesieur, H. \& Blume, S. (1987). The South Oaks Gambling Screen (SOGS): A new instrument for the identification of pathological gamblers. American Journal of Psychiatry, 144(9), 1184-1188. Recuperado de http:// www.ncbi.nlm.nih.gov/pubmed/3631315

Mañoso, V., Labrador, F. \& Fernández-Alba, A. (2004). Tipo de distorsiones cognitivas durante el juego en jugadores patológicos y no jugadores. Psicothema, 16(4), 576-581. Recuperado de http://www.psicothema.com/psicothema. asp?id=3034

National Opinion Center. (1999). Effects of problem gambling. Recuperado de: http://www.lanecounty.org/prevention/gambling/effects_of_PG.htm

Pérez, J. (2012). ¿Cómo regular los casinos? Una propuesta de política pública para mitigar los incentivos perversos de su legislación en México. Revista Legislativa de Estudios Sociales y de Opinión Pública, 5(9), 103-143. Recuperado de www3.diputados.gob.mx/camara/.../Revista-Legislativa-Vol5-Num.9.pdf

Raylu, N. \& Oei, T. (2004). The Gambling Related Cognitions Scale (GRCS): Development, confirmatory factor validation and psychometric properties. Addiction, 99(6), 757-769. Recuperado de http://www.ncbi.nlm. nih.gov/pubmed/15139874

Ruiz, J. \& García, A. (2008). Variables laborales, factores psicosociales y clima familiar en empleados de prisiones. En: Rodríguez, F, et al. (Eds.). Entorno judicial y delincuencia. Oviedo: Universidad de Oviedo. ISBN.: 978-84-8317-707-5., 317-329.

$\boldsymbol{\epsilon} \| 52$ 
Shaffer, H. \& Hall, M. (2001). Updating and refining prevalence estimates of disordered gambling behaviour in the United States and Canada. Canadian Journal of Public Health, 3(92), 168-172. Recuperado de http:// www.ncbi.nlm.nih.gov/pubmed/11496623

Shaw, M. et al. (2007). The effect of pathological gambling on families, marriages, and children. CNS Spectrums, 12(8), 615-622. Recuperado de http://www.ncbi.nlm.nih.gov/pubmed/17667890

Tobon, N., Cano, V. \& Londoño N. (2010). Perfil cognitivo en personas con ludopatía: aproximación a la población no institucionalizada. Revista Virtual Universidad Católica del Norte, 29, 98-121. Recuperado de http:// www.redalyc.org/pdf/1942/194214466007.pdf

Toneatto, T. (1999). Cognitive psychopathology of problem gambling. Substance Use and Misuse, 34(11), 15931604. Recuperado de http://www.ncbi.nlm.nih.gov/pubmed/10468110

Unwin, B., Davis, M. \& De Leeuw, J. (2000). Pathologic gambling. American Family Physician, 61(3), 741-749. Recuperado de http://www.aafp.org/afp/2000/0201/p741.html

Vacca, R. (2005). Aspectos clínicos de la conducta adictiva al juego: una de las adicciones no convencionales. Revista del Instituto de Investigación de Drogodependencias: Salud y drogas, 5(1), 77-97. Recuperado de http://www.redalyc.org/pdf/839/83950105.pdf

Weinstock, J. (2007). Internet gambling is common in college students and associated with poor mental health. The American journal on addictions, 16(5), 325-330. Recuperado de http://www.ncbi.nlm.nih.gov/ pubmed/17882602

Word, S., et al. (2005). Older Adults as Adaptive Decision Makers: Evidence From the lowa Gambling Task. Psychology and Aging, 20(2), 220-225. Recuperado de http://www.ncbi.nlm.nih.gov/pubmed/16029086

\section{Citación}

Iza-Certuche, D. (2015). Presencia de sesgos cognitivos y afectación del clima familiar en jugadores patológicos y no patológicos de la ciudad de Bogotá. Enfoques, 1(2).

Recibido: 09 / 04/ 2015|Aceptado: 11 / 05/ 2015. 